\title{
Nuclear spin relaxation probed by a single quantum dot
}

\author{
A. K. Hüttel ${ }^{1}$, J. Weber ${ }^{1}$, A. W. Holleitner ${ }^{1,2}$, D. Weinmann ${ }^{3}$, K. Eberl ${ }^{4}$, and R. H. Blick ${ }^{1,5}$ \\ ${ }^{1}$ Center for NanoScience and Sektion Physik, Ludwig-Maximilians-Universität, \\ Geschwister-Scholl-Platz 1, 80539 München, Germany \\ ${ }^{2}$ California NanoSystems Institute (CNSI), 4670 Physical Sciences Building North, \\ University of California, Santa Barbara, CA 93106-6105, USA. \\ ${ }^{3}$ Institut de Physique et Chimie des Matériaux de Strasbourg, \\ UMR 7504 (CNRS-ULP), 23 rue du Loess, 67037 Strasbourg, France \\ ${ }^{4}$ Max-Planck-Institut für Festkörperforschung, Heisenbergstraße 1, 70569 Stuttgart, Germany and \\ ${ }^{5}$ Dept. of Electrical 83 Computer Engineering, University of Wisconsin - Madison, \\ 1415 Engineering Drive, Madison, WI 53706-1691, USA.
}

(Dated: June 11, 2003)

\begin{abstract}
We present measurements on nuclear spin relaxation probed by a single quantum dot in a highmobility electron gas. Current passing through the dot leads to a spin transfer from the electronic to the nuclear spin system. Applying electron spin resonance the transfer mechanism can directly be tuned. Additionally, the dependence of nuclear spin relaxation on the dot gate voltage is observed. We find electron-nuclear relaxation times of the order of 10 minutes.

PACS numbers: 73.21.La, 72.25.Rb, 76.60.Es
\end{abstract}

Introduction- The ever increasing demand for computing power as well as theoretical considerations on the basic notions of information processing [1] have led to the development of the new concept of quantum computing 2]. Different experimental systems have been suggested performing quantum computational tasks [3]. Among the most promising of these are quantum dots [4] which can by now be fabricated with great accuracy in a whole variety of circuits enabling not only probing molecular binding mechanisms in coupled dots [5], but also the definition of quantum bits [6, 7].

One of the key questions in quantum information processing is how to efficiently store such quantum bits with a sufficient life time. As suggested by Kane [8] one system for achieving this would be a tunable electron-nuclear spin system, such as a quantum dot coupling to nuclear spins of the embedding crystal matrix. For isolated electron spins trapped in electrostatically defined quantum dots, the theoretical possibilities of realizing qubit operations have already been investigated in great detail [7]. Furthermore, it has been demonstrated that controlled spin transfer between electrons and nuclei is possible in spin polarized two-dimensional [9] and one-dimensional systems [10] and can be detected using electron or nuclear spin resonance techniques 11].

In constrast to these earlier works which beautifully demonstrated tuning of the coupling of a two-dimensional electron system (2DES) to the nuclear spin lattice, as well as controlling and manipulating nuclear spin relaxation [9], we focus on the interaction of electrons confined in a single quantum dot with a much smaller number of nuclei, approaching a mesoscopic regime and strongly localizing the polarization. In addition, we address recent work by Lyanda-Geller et al. 12] who consider nuclear spin relaxation (NSR) caused by a quantum dot coupling to the nuclear magnetic moments. The nuclear system's relaxation time can be several hours, being perfectly suited for phase coherent storage of quantum information. As will be seen below we find according to the predictions of Ref. 12 the relaxation time to depend on the single electron tunneling resonance condition of the dot. We have to note that in our experiment we concentrate on a single quantum dot as compared to recently reported measurements by Ono and Tarucha [13] on a coupled dot.

The main ingredient of our approach is the preparation of a specific quantum dot state, adjacent to a region of spin blockade of transport as discussed earlier [14, 15, 16]. As our measurements in a bi-axial magnet at low fields show, the dot state also possesses a large angular momentum $L$. This effect leads to a spin current, continuously flipping nuclear spins and hence transferring and storing the magnetic momentum through the hyperfine interaction. As expected for this case, electron spin resonance strongly enhances the NSR. This part of the experiment is based on earlier work on photon assisted tunneling in quantum dots 17, 18, 19 and is partly inspired by theoretical work of Engel and Loss [20].

Methods - A typical conductance trace characterizing the quantum dot is shown in Fig. 11 The dot measured here is defined electrostatically in the 2DES of an epitaxially grown $\mathrm{AlGaAs} / \mathrm{GaAs}$ heterostructure: a split gate geometry is written by electron beam lithography on the crystal surface. By negatively charging the gate electrodes, in the 2DES $120 \mathrm{~nm}$ below the surface a quantum dot containing approximately 85 electrons is formed. The data are taken at a bath temperature of $40 \mathrm{mK}$ and an electron temperature of $\sim 80 \mathrm{mK}$ in a ${ }^{3} \mathrm{He} /{ }^{4} \mathrm{He}$ dilution refrigerator system. A similar conductance pattern as in Fig. 1 was obtained in our earlier work on spin blockade in a dot containing about 50 electrons $[16]$. At $4.2 \mathrm{~K}$ the carrier density of the 2DES is $1.8 \times 10^{15} \mathrm{~m}^{-2}$ and the 


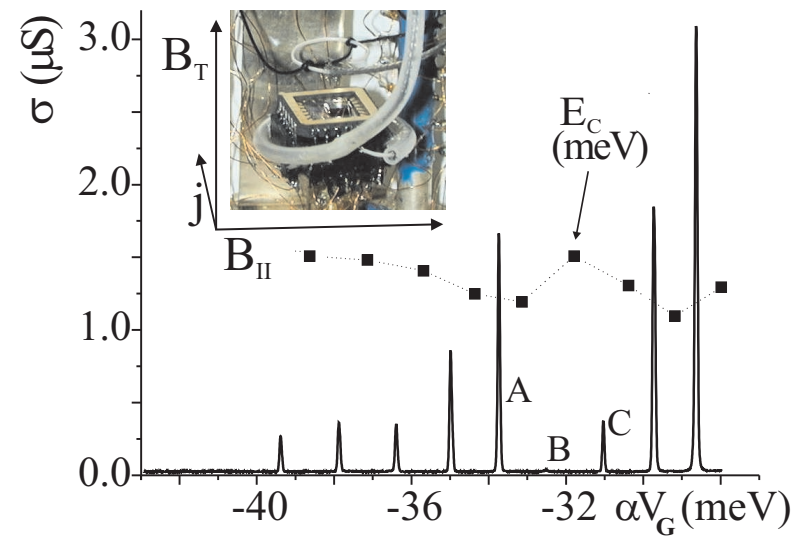

FIG. 1: Coulomb blockade oscillations of conductance $\sigma=$ $d I / d V$ of a single quantum dot vs. gate voltage $V_{G}$. The dotted line gives the charging energy $E_{C}$ for adding single electrons. As seen the energy assumes a local maximum between peak B and C. Inset: sample holder setup including radiowave and microwave antennae. The $\mathrm{AC}$ signal in the antennae leads to an alternating magnetic field perpendicular to the sample's surface.

electron mobility is $75 \mathrm{~m}^{2} / \mathrm{Vs}$. For DC measurements, a source-drain voltage of $20 \mu \mathrm{V}$ is provided. The addition energy of the dot is given by $E_{C} \sim 1.25 \mathrm{meV}$, as indicated by the dotted line in Fig. 1.

Experiment- In the conductance spectrum of Fig. 1] a sequence of three peaks is marked by the letters $\mathrm{A}, \mathrm{B}, \mathrm{C}$. Peak A displays conventional conductance, whereas peak $\mathrm{B}$ is nearly completely blocked at low transport voltage and peak $\mathrm{C}$ shows a response smaller than average. In subsequent measurements, the suspended loop antenna visible in the inset of Fig. 1 is emitting microwave radiation onto the sample chip: Fig. 2(a) again displays the three peaks now showing the induced photocurrent under irradiation at $10.01 \mathrm{GHz}$. It is important to note that prior to taking these data traces the perpendicular magnetic field was ramped from $B_{\perp}=0 \mathrm{~T}$ up to $B_{\perp, \max } \approx 0.5 \mathrm{~T}$ in $t_{r}=11 \mathrm{~min}$, maintained at this value for $t_{m}=8 \mathrm{~min}$, and subsequently reduced to $B_{\perp}=0 \mathrm{~T}$ within 11 min. As seen peak A gives the conventional rectification signal with a forward and backward pumped current [21]. Surprisingly, the spin blockade transition at $\mathrm{B}$ reveals a backward current only. This can be explained by strongly differing excitation energies of the quantum dot at subsequent electron numbers, consistent with level scrambling causing spin blockade type-II.

The assumption of a certain spin texture gains evidence when focussing on resonance $\mathrm{C}$ - which, being located next to the spin blocked peak, can feature a high spin as well: after ramping $B_{\perp}$ the relaxation of the current trace requires additionally more than $10 \mathrm{~min}$. Testing the available parameter ranges, we found $B_{\perp, \max }=$ $40 \mathrm{mT}$, as well as ramp times and a waiting period of

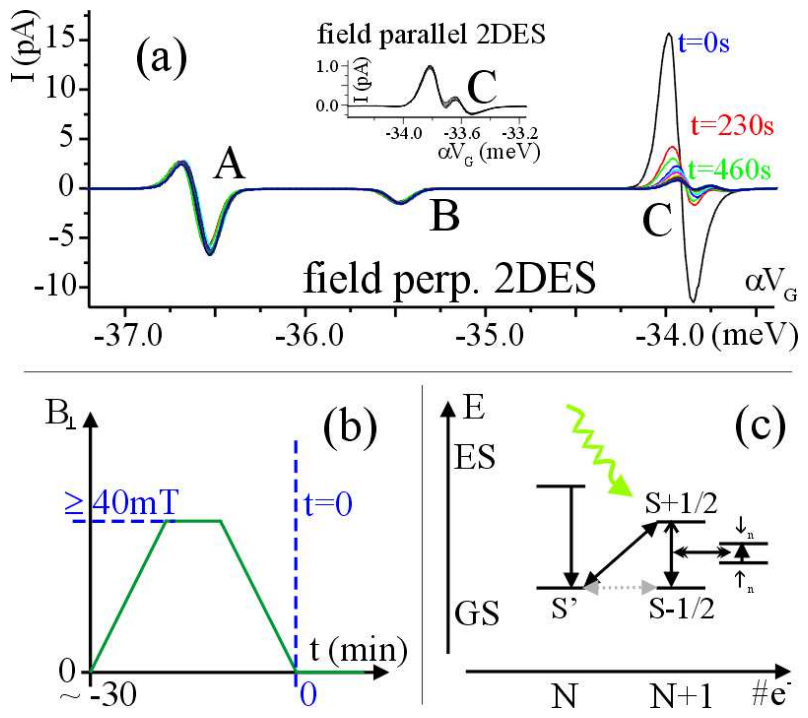

FIG. 2: (a) Quantum dot photocurrent peaks A, B, and C under microwave radiation at $10.01 \mathrm{GHz}$. Prior to taking these data the perpendicular magnetic field was ramped from $B_{\perp}=0 \mathrm{~T}$ to $487 \mathrm{mT}$ in $t_{r} \sim 11 \mathrm{~min}$, maintained at this value for $t_{m} \sim 8 \mathrm{~min}$, and reduced to $B_{\perp}=0 \mathrm{~T}$ within $11 \mathrm{~min}$. A strong memory effect at peak $\mathrm{C}$ can be observed. The inset gives the same measurement for peak $\mathrm{C}$, using a parallel field orientation. Obviously no long-term memory is found. (b) Schematic plot detailing $B(t)$ in the measurement setup. (c) Level diagram for the transition from $N$ to $(N+1)$ electrons at peak $\mathrm{C}$ (see text for further details).

$t_{r / m}=6$ min to be sufficient for clearly demonstrating the effect.

This time dependence is attributed to the observation of a slowly decaying nuclear spin polarization, which has been induced during the magnetic field sweep by dynamic polarization processes. Here, the accessibility of transport channels depends on the population of spin states [9, 10]. In a quantum dot in an AlGaAs/GaAsheterostructure with a diameter and height of $125 \mathrm{~nm}$ and $10 \mathrm{~nm} \tilde{N} \sim 2.2 \times 10^{7}$ nuclei are engulfed by the electronic volume. For comparison, a rough estimate gives $10^{8}-10^{9}$ electrons passing the dot during the magnetic field sweep of $30 \mathrm{~min}$. In addition, a completely polarized nuclear spin population has been shown to give rise to local magnetic fields of up to several Tesla [9, 22]. Even partial polarization or polarization within a small volume is expected to have a clearly visible effect.

The inset in Fig. 2(a) gives an identical measurement for peak $\mathrm{C}$ in a cycled magnetic field parallel to the 2DES - as seen no memory effect is observed. This leads us to the conclusion that orbital effects bound to a particular spin state are responsible for coupling to the nuclear magnetic moments. A pure spin flip would obey Zeeman splitting in a parallel magnetic field as well, and the phenomenon should persist in this case. In an intuitive picture, at peak $\mathrm{C}$ the electrons tunneling through 
the quantum dot can be thought to be passing through a high- $L$ state, circulating at the edge and allowing to transfer momentum from the electronic to the nuclear system.

A possible level scenario of the spin flip operation is given in the diagram of Fig. 2(c): as measured, we assume the direct transition probability between the $N$ and $(N+1)$ electron ground states to be low; single electron tunneling is partly suppressed. An increase in current via the excited $(N+1)$ electron state takes place as soon as irradiation enhances the energy available. Relaxation into the ground state via hyperfine coupling to the nuclear spin system comprises a change in spin quantum number by $\Delta S=1$, spin conservation in the hyperfine interaction results in a flip-flop process of electron and nuclear spins 10]. This brings the spin of a nearby nucleus from $\left|\downarrow_{n}\right\rangle$ into the state $\left|\uparrow_{n}\right\rangle$. The dot remains in the $(N+1)$ electron ground state until the electron tunnels out via the ground state transition and the cycle restarts. On the timescale given, the spin flip rate required for polarization is consistent with theoretical predictions for a similar quantum dot 24], where the energy mismatch between electronic and nuclear Zeeman splitting, otherwise suppressing this process, is compensated by phonon emission.

However, the relaxation by flip-flop processes is only possible as long as sufficient nuclei with appropriate spin direction are available. Assuming a nonzero polarization, hyperfine relaxation decreases, and the trapping effect described above is deactivated, leading to an increase in current. This gives a possible mechanism of detection of the gradual depolarization after ramping down the magnetic field. Other mechanisms include a shift of electronic levels induced by a remaining effective nuclear magnetic field. The quantum dot operates as partial spin filter and inverter; a weak polarization of the nuclear spins even without supporting microwave radiation is possible, as long as a magnetic field perpendicular to the surface provides an orientation.

Subsequently we want to address the change in nuclear relaxation time in dependence of the quantum dot's resonance state as Lyanda-Geller et al. investigated in their calculations 12]: Again we focus on resonance $\mathrm{C}$ in a perpendicular field orientation with the field cycling as introduced above. The main difference now is that relaxation of the photocurrent trace after switching off $B_{\perp}$ is not monitored sweeping continuously over the gate voltage range. A first current trace is recorded; then the gate voltage is kept either at SET resonance $V_{g}^{\text {res }}$ or off resonance $V_{g}^{\text {off }}$, as shown schematically in the insets of Fig. 3] 10 min later, an additional trace of the peak is taken. Obviously, in the case of SET resonance the relaxation slows down considerably. As shown by the authors of Ref. [12], a non-negligible spin-orbit interaction 16 in combination with the differing nature of coupling processes in separate gate voltage regimes causes such behaviour.

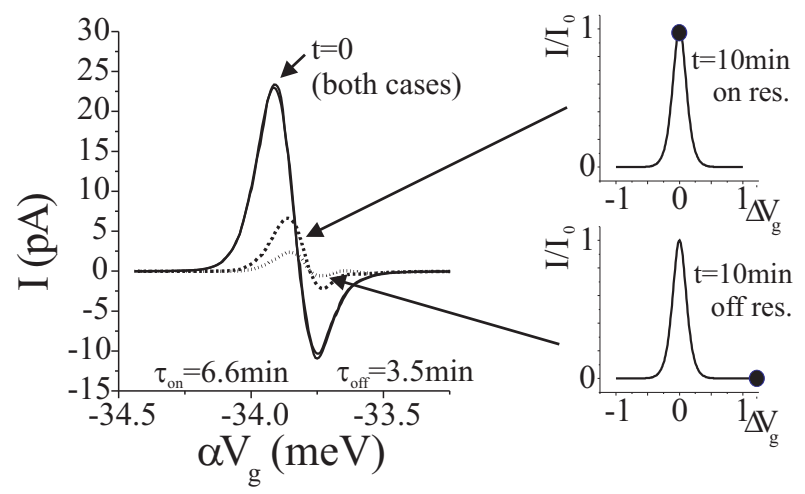

FIG. 3: Dependence of NSR on Coulomb blockade: after cycling $B_{\perp}$ as described in the text, and taking a trace for reference at $t=0$ (solid lines) the gate voltage is kept fixed for a waiting period of $\Delta t=10 \mathrm{~min}$. Relaxation is then determined in dependence of the gate voltage position. Relaxation times strongly vary comparing waiting positions in resonance and off resonance.

In analyzing the relaxation process quantitatively we compare the integrated difference of relaxed and excited photocurrent traces and finally normalize it with respect to the relaxed curve. This is defined by a function

$$
E[t] \equiv \frac{\int_{V_{G 1}}^{V_{G 2}} d V_{G}\left|I\left(V_{G}, t\right)-I\left(V_{G}, \infty\right)\right|}{\int_{V_{G 1}}^{V_{G 2}} d V_{G}\left|I\left(V_{G}, \infty\right)\right|}
$$

The characteristic decay time constant of $E[t]$ corresponds to the nuclear spin relaxation time $T_{1}$ and typically assumes values of $\tau \sim 5 \ldots 12 \mathrm{~min}$. In the measurement described above, we find as relaxation times for $E[t]$ depending on the gate voltage during waiting $\tau_{\text {res }}=6.6 \mathrm{~min}$ and $\tau_{\text {off }}=3.5 \mathrm{~min}$, hence again supporting the theoretical assumptions of Lyanda-Geller et al.

In extending the discussion above we now can apply classical electron spin resonance to tune the nuclear relaxation time. This is performed by again irradiating at $10.01 \mathrm{GHz}$ and measuring $E[t]$ for different values of an additional parallel magnetic field which couples to the spin only. The perpendicular field is sequentially polarizing the nuclear spins through the quantum dot at $200 \mathrm{mT}$. The maximal amplitude at $t=0$, i.e. directly after the external field $B_{\perp}$ has been brought to zero, is given by $E[0]$ and corresponds to maximal polarization of the nuclear spin system within our interval of observation. $E[t]$ then decays exponentially, as can be seen in the exemplary plot of Fig. 4(a). In Fig. 4(b) at $10.01 \mathrm{GHz}$ we obviously find for a field of $B_{\|}=1.7 \mathrm{~T}$ a clear resonant feature in the NSR time which agrees with the value obtained from $E_{Z}=g \mu_{B} B$ assuming $g=-0.42$. Particularly, the life time reduction in resonance supports the level diagram sketched in Fig. 2(c): the electron spin res- 

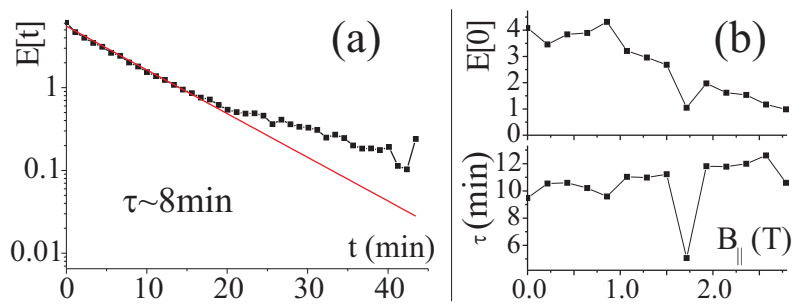

FIG. 4: (a) Exemplary plot of the relaxation of peak $\mathrm{C}$ as seen in Fig. 2(a) with a relaxation constant of $T_{1} \sim \tau \sim 8 \mathrm{~min}$. The function $E[t]$ as a measure for the peak relaxation and thereby the nuclear spin polarization is defined in the text. (b) Electron spin resonance (ESR) in an additional constant parallel magnetic field found by comparing the maximal polarization $E[t=0]$ and the relaxation times $\tau$. Mixing of ground and excited states leads to a strongly reduced $\tau$ and $E[0]$.

onance leads to a mixing of the ground and excited states with a spin change of $\Delta S=1$. This it to be considered as bypassing the pumping of nuclear spins through an electron spin transition. As seen we are able to achieve a change of over $50 \%$ in NSR time.

Conclusions - Relating to SET blockade regimes in a single quantum dot we find strong coupling of electron and nuclear spins via the hyperfine interaction. This leads to measured nuclear relaxation times exceeding $10 \mathrm{~min}$. In accordance with reference [12] we find that NSR is maximal in the regime of Coulomb blockade. Electron spin resonance is applied to broadly vary NSR. As we observe electron-nuclear spin coupling at moderate fields of some $50 \mathrm{mT}$ in conjunction with the tuning mechanisms introduced, we conclude that - although in our case still a large number of nuclei is addressed simultaneously - this will strongly support quantum information processing in solid state systems, being a first step towards quantum state transfer and the long-term storage of quantum spin information.

Acknowledgements - We like to thank J.P. Kotthaus, R. Gross, and R. Jalabert for detailed discussions and A. Kriele and K. Neumaier for expert technical help. We acknowledge financial support by the Deutsche Forschungsgemeinschaft through the Schwerpunkt 'Quanteninformationsverarbeitung' (Bl/487-2-2) and the Defense Advanced Research Projects Agency (EOARD project: F61775-01-WE037). AKH gratefully acknowledges support by the Studienstiftung des deutschen Volkes.

[1] R. Landauer, Phys. Lett. A 217, 188 (1996).
[2] M. A. Nielsen and I. L. Chuang, Quantum Computation and Quantum Information, (Cambridge University Press 2000).

[3] D. Bouwmeester, A. Ekert, and A. Zeilinger (eds.), The physics of quantum information: Quantum Cryptography, Quantum Teleportation, Quantum Computation (Springer 2000); A. Barenco, D. Deutsch, A. Ekert, and R. Jozsa, Phys. Rev. Lett. 74, 4083 (1995).

[4] L. P. Kouwenhoven, C. M. Marcus, P. L. McEuen, S. Tarucha, R. M. Westervelt, and N. S. Wingreen, in Mesoscopic Electron Transport, edited by L. L. Sohn, L. P. Kouwenhoven, and G. Schön, series E, vol. 345, p. 105, Kluwer Dordrecht, Netherlands (1997); R. Ashoori, Nature 379, 413 (1996).

[5] A. W. Holleitner, R. H. Blick, A. K. Hüttel, K. Eberl, and J. P. Kotthaus, Science 297, 70 (2002).

[6] R. H. Blick and H. Lorenz, Proc. IEEE Int. Symp. Circ. Sys. II-245, 28 (2000).

[7] D. Loss and D. P. DiVincenzo, Phys. Rev. A 57, 120 (1998); P. Recher, E. V. Sukhorukov, and D. Loss, Phys. Rev. Lett. 85, 1962 (2000); Xuedong Hu and S. Das Sarma, Phys. Rev. A 64, 042312 (2001).

[8] B. E. Kane, Nature 393, 133 (1998).

[9] J. H. Smet, R. A. Deutschmann, F. Ertl, W. Wegscheider, G. Abstreiter, K. v. Klitzing, Nature 415, 281 (2002).

[10] K. R. Wald, L. P. Kouwenhoven, P. L. McEuen, N. C. van der Vaart, and C. T. Foxon, Phys. Rev. Lett. 73, 1011 (1994).

[11] M. Dobers, K. v. Klitzing, J. Schneider, G. Weimann, and K. Ploog, Phys. Rev. Lett. 61, 1650 (1988); S. Kronmüller, W. Dietsche, K. v. Klitzing, G. Denninger, W. Wegscheider, and M. Bichler, Phys. Rev. Lett. 82, 4070 (1999).

[12] Y. B. Lyanda-Geller, I. L. Aleiner, and B. L. Altshuler, Phys. Rev. Lett. 89, 107602 (2002).

[13] K. Ono and S. Tarucha, Proc. of the 26. Int. Conf. Semic., H254 (2002).

[14] L. P. Rokhinson, L. J. Guo, S. Y. Chou, and D. C. Tsui Phys. Rev. B 63, 035321 (2001).

[15] D. Weinmann, W. Häusler, and B. Kramer, Phys. Rev. Lett. 74, 984 (1995); Ann. Phys. (Leipzig) 5, 652 (1996).

[16] A. K. Hüttel, H. Qin, A. W. Holleitner, R. H. Blick, K. Neumaier, D. Weinmann, K. Eberl, and J. P. Kotthaus, Europhys. Lett. 62, 712 (2003); cond-mat/0109014

[17] L. P. Kouwenhoven, S. Jauhar, J. Orenstein, P. L. McEuen, Y. Nagamune, J. Motohisa, and H. Sakaki Phys. Rev. Lett. 73, 3443 (1994).

[18] R. H. Blick, D. W. van der Weide, R. J. Haug, K. v. Klitzing, and K. Eberl, Appl. Phys. Lett. 67, 3924 (1995).

[19] R. H. Blick, V. Gudmundsson, R. J. Haug, K. v. Klitzing, and K. Eberl, Phys. Rev. B 57, R12685 (1998).

[20] H.-A. Engel and D. Loss, Phys. Rev. Lett. 86, 4648 (2001).

[21] J. Weis, R. J. Haug, K. v. Klitzing, and K. Ploog, Semicond. Sci. Technol. 10, 877 (1995).

[22] D. Paget, G. Lampel, B. Sapoval, and V. I. Safarov, Phys. Rev. B 15, 5780 (1977).

[23] A. V. Khaetskii, D. Loss, and L. Glazman, Phys. Rev. Lett. 88, 186802 (2002).

[24] S. I. Erlingsson, Y. V. Nazarov, and V. I. Fal'ko, Phys. Rev. B 64, 195306 (2001). 